\title{
SOLAR SAIL ORBIT OPERATIONS AT ASTEROIDS: EXPLORING THE COUPLED EFFECT OF AN IMPERFECTLY REFLECTING SAIL AND A NONSPHERICAL ASTEROID *
}

\author{
Esther Morrow ${ }^{\dagger}$ \\ D. J. Scheeres ${ }^{\ddagger}$ \\ Dan Lubin $\S$
}

May 16, 2002

\begin{abstract}
$\underline{\text { Abstract }}$
This paper continues the work of previous studies which examined solar sail dynamics about an asteroid. In the current study the effect of an imperfectly reflecting sail on the spacecraft dynamics is evaluated and the effect of a non-spherical asteroid is explored. The effect of the imperfect sail is seen to cause a diminished solar radiation pressure force along the sun line and modifies the possible hovering locations. The effect of a non-spherical asteroid is modeled by using the $J_{2}$ gravity field contribution. We find explicit predictions for the coupling between the (assumed dominant) solar radiation pressure and the gravity field perturbation. Although the special class of stable orbits for solar sails are generally stable under the $J_{2}$ perturbation, we find certain parameter combinations that may lead to instabilities in the sail orbit. The analytical work is verified by numerical computations.
\end{abstract}

\section{Introduction}

The natural appeal of solar sailing coupled with the development of microtechnologies and new materials has recently caused increased interest in this field. At the same time, we are just beginning to study asteroids in situ. Because solar sail spacecraft have an inexhaustable fuel supply from sunlight, they are capable of performing long term, multiple objective missions to asteroids, significantly decreasing the time between missions. They are also

\footnotetext{
* Copyright (C)2002 The American Institute of Aeronautics and Astronautics Inc. All rights reserved. AIAA Paper 20024991

$\dagger^{\dagger}$ Research Associate, California Space Institute, Scripps Institution of Oceanography, University of California, San Diego, La Jolla, CA

$\ddagger$ Assistant Professor, Department of Aerospace Engineering, The University of Michigan, Ann Arbor, MI

$\S$ Associate Research Physicist, California Space Institute, Scripps Institution of Oceanography, University of California, San Diego, La Jolla, CA
}

capable of orbiting asteroids or hovering over the surface for extended periods of time which allows surface mapping or sample returns.

In our first paper ${ }^{1}$, we examined the feasibility of both asteroid orbits and equilibrium points, making many assumptions on both the spacecraft and asteroid, i.e., that the spacecraft is perfectly reflecting and planar and that the asteroid is a point mass in a circular orbit about the sun. In our current analysis we relax some of these assumptions in an effort to move toward a more realistic model of sail motion about an asteroid.

We change the sail from being perfectly reflecting to imperfectly reflecting. Ideally, all photons incident on the sail will be reflected. In reality, however, some of the photons will be absorbed into the sail material. The efficiency of the sail will decrease depending on the materials used. For the sake of this paper, we are assuming $85 \%$ reflectivity, which is a conservative estimate. Materials are available now which can achieve about $90 \%$ reflectivity. ${ }^{2}$

We also generalize the asteroid gravity field from a point mass to an oblate spheroid with its rotation axis tilted away from the asteroid orbit angular momentum. The asteroid spin axis is fixed in inertial space. Since the asteroid spin pole is about the symmetry axis, we do not consider the asteroid spin rate. Though we add in perturbations due to oblateness, we still neglect perturbations due to asteroid ellipticity. We make this assumption since the largest perturbation comes from the $J_{2}$ effect and because the sail will probably be placed in an orbit which minimizes the effect of the asteroid ellipticity in general.

\section{Imperfectly reflecting sail model}

When taking an imperfectly reflecting solar sail into consideration, the solar radiation pressure (SRP) acceleration vector is no longer strictly nor- 
mal to the sail. The equation for SRP for a perfectly reflecting sail is $\mathbf{a}=a_{p}(\mathbf{l} \cdot \mathbf{n})^{2} \mathbf{n}$. For an imperfectly reflecting sail we must include a component of force in the transverse direction to the sail normal due to absorption of photons:

$$
\begin{aligned}
\mathbf{a}= & {\left[a_{x} a_{y} a_{z}\right] } \\
= & \frac{1}{2} a_{n p}(1+\eta)(\mathbf{1} \cdot \mathbf{n})^{2} \mathbf{n} \\
& +\frac{1}{2} a_{n p}(1-\eta)(\mathbf{1} \cdot \mathbf{n})(\mathbf{1} \cdot \mathbf{t}) \mathbf{t}
\end{aligned}
$$

or

$$
\begin{aligned}
a_{x}= & \frac{1}{2} a_{n p}(1+\eta) \cos ^{3} \phi \cos ^{3} \alpha \\
& +\frac{1}{2} a_{n p}(1-\eta) \cos ^{3} \phi \cos \alpha \sin ^{2} \alpha \\
a_{y}= & \frac{1}{2} a_{n p}(1+\eta) \cos ^{2} \phi \sin \phi \cos ^{3} \alpha \\
& +\frac{1}{2} a_{n p}(1-\eta) \cos ^{2} \phi \sin \phi \cos \alpha \sin ^{2} \alpha \\
a_{z}= & \frac{1}{2} a_{n p}(1+\eta) \cos ^{2} \phi \cos ^{2} \alpha \sin \alpha \\
& -\frac{1}{2} a_{n p}(1-\eta) \cos ^{2} \phi \cos ^{2} \alpha \sin \alpha
\end{aligned}
$$

where $a_{n p}$ is related to sail acceleration $a_{p}$ by

$a_{n p}=\frac{2}{(1+\eta)} \frac{\sqrt{1+\tan ^{2} \zeta}}{(1-\tan \xi \tan \zeta)^{2}} a_{p}$

and $\alpha$ is the pitch angle made up of two components, the center line angle $\zeta$ and the cone angle $\xi ; \phi$ is the sail roll angle; and $\eta$ is the sail reflectivity value, $\mathbf{l}$ is the unit vector of incoming sunlight, $\mathbf{n}$ is the unit normal to the sail and $\mathbf{t}$ is the unit vector transverse to the normal.

It can be seen that, although the tranverse component must be taken into account when the sail is at an angle with respect to the sun-line, it makes no difference in the direction of the normal force when the sail faces the sun. In this case, the normal force is simply reduced by a factor of $(1+\eta) / 2$.

\section{Equilibrium points with an imperfectly reflecting sail about point-mass asteroids}

Since McInnes ${ }^{2}$ has already studied the effect of an imperfectly reflecting sail on equilibrium points about a point-mass Earth, we will not discuss equilibruim points about point-mass asteroids in much detail. Even though the gravitational effect of an asteroid is much less than that of the Earth, the effect scales proportionately. Our conclusions are the same as McInnes', i.e., that the direction in which the SRP force vector can be oriented is limited by the change in the SRP normal force acting on the sail. Figure 1 is a two-dimensional plot of possible equilibrium points about a point-mass asteroid. Each line in the plot represents a continuum of possible equilibrium points for a given amount of normalized sail acceleration. This plot is similar to that found by McInnes ${ }^{2}$. Note that the volume of space where equilibrium points are possible is significantly decreased from the volume of space found with a perfectly reflecting sail spacecraft.

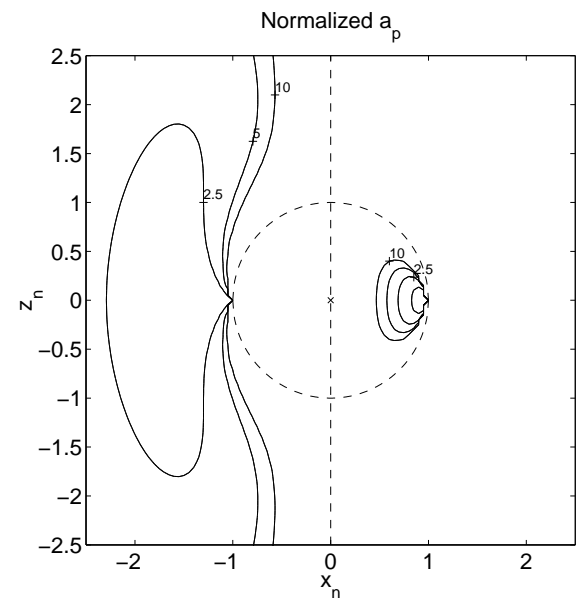

Figure 1: Contour plot of equilibrium points about a point-mass asteroid with an imperfectly reflecting sail. Dashed lines represent the Hill radius and show the "terminator" line of asteroid.

In Figure 1, the graph is in the $x z$-plane of the rotating frame but position coordinates have been normalized by $x_{n}=r_{n} \cos \theta \cos \psi$ and $z_{n}=r_{n} \sin \theta$ where $r_{n}$ is the distance between the spacecraft and the asteroid normalized by the Hill radius, $r / r_{H}$. The Hill radius is given by

$r_{H}=\left(\mu / 3 N^{2}\right)^{1 / 3}$

and marks a boundary $\left(r_{n}=1\right)$ where the sail may and may not fly. In this equation, $\mu$ is the asteroid gravitational parameter and $N$ is asteroid orbit angular velocity. On the day side of the asteroid, the sail may not fly inside the Hill radius. It also may not fly on the outside of the boundary on the night side of the asteroid. The sail acceleration $a_{n p}$ has been normalized by $\mu / r_{H}^{2}$

The end result of having an imperfectly reflecting sail is that possible equilibrium points for a sail with a given characteristic acceleration are pushed radially away from the asteroid. The acceleration in the radial direction is decreased, therefore, the sail must move outward in order to compensate. 


\section{Oblate asteroid model}

In this section we consider the effect of asteroid oblateness on the dynamics of a solar sail. We will model our oblate asteroid as a body with semi-major axes $a \geq b=c$ which is spinning at a constant rate about its smallest axis $c$.

Figure 2 shows the orientation of an oblate asteroid in an inertial frame $(X, Y, Z)$. The asteroid spins about the $\mathbf{p}_{z}$-axis, whose orientation is given by the angles $\tilde{\alpha}$ and $\delta$. The oblateness assumption is reasonable for some of the larger asteroids in the solar system, and, even for irregularly shaped asteroids, represents the first non-trivial force perturbation (beyond SRP) acting on the sail's orbit.

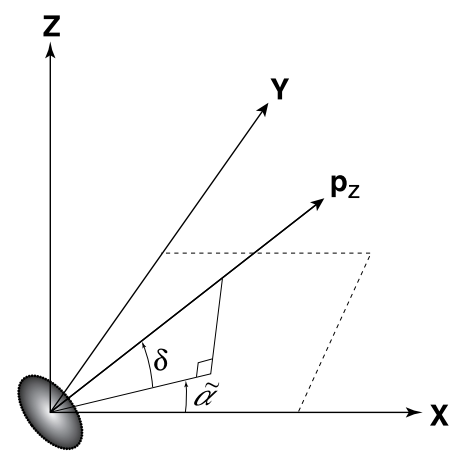

Figure 2: The $\mathbf{p}_{z}$-axis is fixed in the inertial frame $(X, Y, Z)$. Its coordinates are given by the Euler angles $\tilde{\alpha}$ and $\delta$.

The unit vector of the asteroid's rotation axis is described in inertial coordinates as

$\hat{\mathbf{p}}_{z}=[\cos \delta \cos \tilde{\alpha} \cos \delta \sin \tilde{\alpha} \sin \delta]$

where $\delta$ is the declination of the asteroid rotation pole and $\tilde{\alpha}$ is the right ascension of the asteroid rotation pole in inertial space. The spacecraft orbit is described by

$\mathbf{r}_{h}=\sin \Omega \sin i \hat{x}-\cos \Omega \sin i \hat{y}+\cos i \hat{z}$

$\mathbf{r}_{T}=-\sin \Omega \cos i \hat{x}-\cos \Omega \cos i \hat{y}+\sin i \hat{z}$

$\mathbf{r}_{\Omega}=\cos \Omega \hat{x}+\sin \Omega \hat{y}$

where $i$ is the orbit inclination and $\Omega$ is the longitude of ascending node relative to inertial space.

$\mathbf{r}=r \mathbf{r}_{s}$

is the position vector from the asteroid to the spacecraft where the unit vector is given by

$\mathbf{r}_{s}=\cos u \mathbf{r}_{\Omega}+\sin u \mathbf{r}_{T}$

where $u=\omega+\nu, \omega$ is the spacecraft orbit argument of periapsis and $\nu$ is spacecraft true anomaly.
For detailed discussion we will use a rotating coordinate frame fixed with respect to the asteroid-sun line, denoted as $(x, y, z)$. We assume that the $z$ axes in the rotating and inertial frames are aligned.

While the asteroid rotation pole is fixed in the inertial frame, it rotates in the rotating frame and is described by:

$\hat{\mathbf{p}}_{z}=\cos \delta \cos N t \hat{\mathbf{x}}-\cos \delta \sin N t \hat{\mathbf{y}}+\sin \delta \hat{\mathbf{z}}$

where the $N t$ term denotes the polar angle of the asteroid about the sun. Note that we assume a circular orbit for the asteroid, although this assumption can be relaxed without fundamentally changing the results of this paper.

With this definition the $J_{2}$ force potential with a tilted rotation axis is given $b^{3}$ :

$U_{J_{2}}=\frac{\mu J_{2}}{2 r^{3}}\left[1-\frac{3}{r^{2}}\left(\mathbf{r} \cdot \hat{\mathbf{p}}_{z}\right)^{2}\right]$

where $\mathbf{r}$ is the position vector from the asteroid to the sail, and is specified as

$\mathbf{r}=r[\cos \theta \cos \psi \hat{\mathbf{x}} \cos \theta \sin \psi \hat{\mathbf{y}} \sin \theta \hat{\mathbf{z}}]$

where $\theta$ is the declination angle measured from the $x y$-plane toward the $z$-axis and $\psi$ is the right ascension angle measured in the $x y$-plane from the $+x$ axis, as shown in Figure 3.

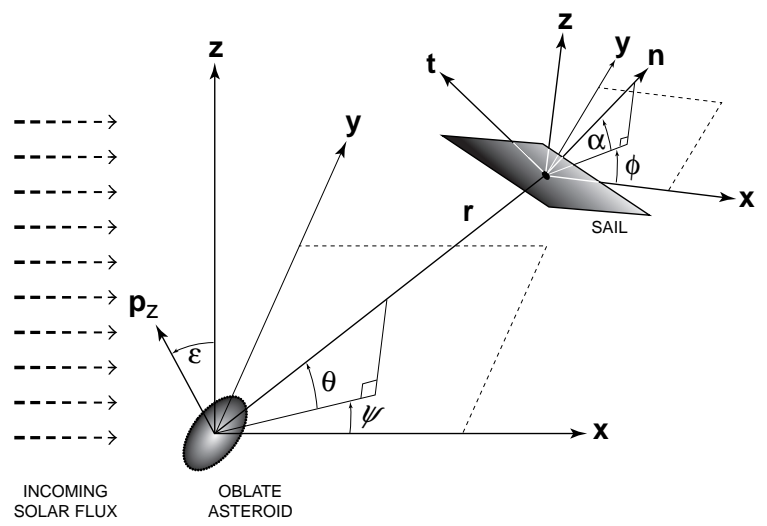

Figure 3: Solar sail near an oblate asteroid with asteroid rotation about $\mathbf{p}_{z}$-axis. The vector $\mathbf{p}_{z}$ is fixed in inertial space while the asteroid revolves about the sun.

The gravitational attraction is computed as:

$$
\begin{aligned}
\frac{\partial U}{\partial \mathbf{r}}= & -\frac{3}{2} \frac{\mu J_{2}}{r^{5}} \mathbf{r}+\frac{15}{2} \frac{\mu J_{2}}{r^{7}}\left(\mathbf{r} \cdot \hat{\mathbf{p}}_{z}\right)^{2} \mathbf{r} \\
& -3 \frac{\mu J_{2}}{r^{5}}\left(\mathbf{r} \cdot \hat{\mathbf{p}}_{z}\right) \hat{\mathbf{p}}_{z}
\end{aligned}
$$


Incorporating $\partial U / \partial \mathbf{r}$ into the equations of motion in the rotating coordinate frame ${ }^{1}$, we have:

$$
\begin{aligned}
\ddot{x}-2 N \dot{y}= & -\frac{\mu}{r^{3}} x+3 N^{2} x-\frac{3}{2} \frac{\mu J_{2}}{r^{5}} x \\
& +\frac{15}{2} \frac{\mu J_{2}}{r^{7}}\left(\mathbf{r} \cdot \hat{\mathbf{p}}_{z}\right)^{2} x \\
& -3 \frac{\mu J_{2}}{r^{5}}\left(\mathbf{r} \cdot \hat{\mathbf{p}}_{z}\right) \cos \delta \cos N t+a_{x} \\
\ddot{y}+2 N \dot{x}= & -\frac{\mu}{r^{3}} y-\frac{3}{2} \frac{\mu J_{2}}{r^{5}} y+\frac{15}{2} \frac{\mu J_{2}}{r^{7}}\left(\mathbf{r} \cdot \hat{\mathbf{p}}_{z}\right)^{2} y \\
& +3 \frac{\mu J_{2}}{r^{5}}\left(\mathbf{r} \cdot \hat{\mathbf{p}}_{z}\right) \cos \delta \sin N t+a_{y} \\
\ddot{z}= & -\frac{\mu}{r^{3}} z-N^{2} z-\frac{3}{2} \frac{\mu J_{2}}{r^{5}} z \\
& +\frac{15}{2} \frac{\mu J_{2}}{r^{7}}\left(\mathbf{r} \cdot \hat{\mathbf{p}}_{z}\right)^{2} z \\
& -3 \frac{\mu J_{2}}{r^{5}}\left(\mathbf{r} \cdot \hat{\mathbf{p}}_{z}\right) \sin \delta+a_{z}
\end{aligned}
$$

where the origin of the rotating reference frame is centered at the asteroid with the positive $x$-axis in the anti-solar direction, the $z$-axis normal to the ecliptic, and the $y$-axis according the the right hand rule. This frame rotates about the $z$-axis with angular velocity $N=\sqrt{\mu_{\text {sun }} / a_{\text {ast }}^{3}}$, where $\mu_{\text {sun }}$ is the sun's gravitational parameter $\left(\sim 1.34 \times 10^{11}\right.$ $\mathrm{km}^{3} / \mathrm{s}^{2}$ ), and $a_{\text {ast }}$ is the asteroid's heliocentric orbit radius in $\mathrm{km}$. The gravitational parameter of the asteroid is denoted as $\mu$. The entire sail-asteroid model is shown in Figure 3. The sail position coordinates are determined by $r, \theta$ and $\psi$ in the rotating frame $(\mathbf{x}, \mathbf{y}, \mathbf{z})$. The sail orientation is given by pitch angle $\alpha$ and roll angle $\phi$. Force vectors are along the sail normal (n) and transverse to the sail (t).

\section{Sail dynamics about an Oblate Spheroid}

We now consider the effect of asteroid oblateness on the dynamics of our sail orbit. First we review some of the basic results originally reported in (Morrow et al. $)^{1}$. In that study we found a class of frozen orbits about the asteroid which were stable in the sense that they neither impacted the asteroid nor escaped. We found an upper bound on sail acceleration at $1 \mathrm{AU}$ to be

$a_{p 0}^{M}=(\tilde{R} / 16)\left(\mu / r_{0}^{2}\right)$

where $\tilde{R}$ is the asteroid-sun distance in $\mathrm{AU}$ and $r_{0}$ is the mean asteroid radius. We showed that a sufficient condition for an orbit to be bound was that

$a_{s c}<(\tilde{R} / 4) \sqrt{\mu / a_{p 0}}$ where $a_{s c}$ is the spacecraft semi-major axis. Bound orbits were close to a family of frozen orbits defined for these systems with eccentricity close to 0 , sunsynchronous, with orbit plane in the sun plane of sky, and with constant semi-major axis on average. The initial conditions for these stable orbits were $e \sim 0$, $i=\pi / 2, \omega= \pm \pi / 2$ and $\lambda \sim \mp \pi / 2$. With these initial conditions the eccentricity and longitude of the ascending node were bound.

Orbit about Ida with imperfect sail in rotating frame

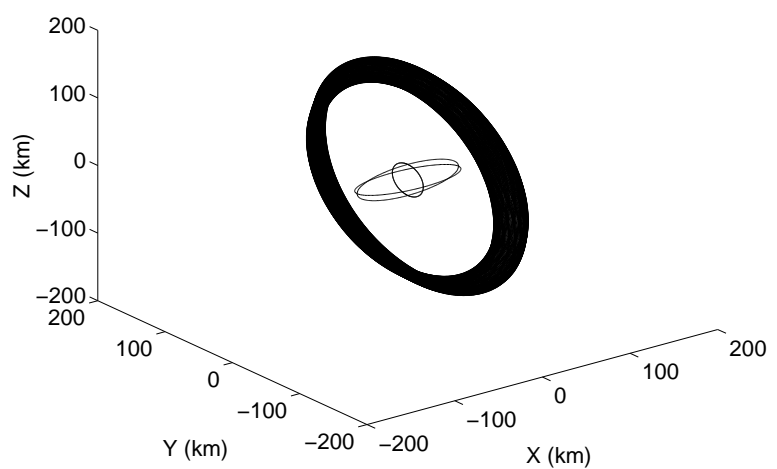

Figure 4: Orbit about Ida in the rotating reference frame with $J_{2}=0$. The orbit is stable in the sense that it neither crashes nor escapes.

Orbit about Ida with imperfect sail \& J2 effect

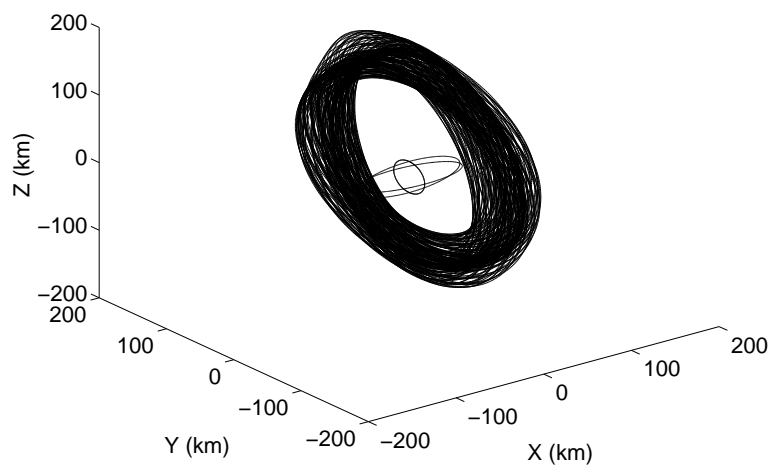

Figure 5: Orbit about Ida with $J_{2} \neq 0$. All other conditions are the same as in figure 4 .

In our current analysis we will concentrate on one specific case, that of sail motion about a suitably simplified model of the asteroid Ida. In Figures 4 and 5 we show numerically integrated trajectories about our Ida model, one including $J_{2}$ and one not including it. For both cases the model parameters are $a_{p o}$ $=.5 \mathrm{~mm} / \mathrm{s}^{2}$ and the sail is imperfectly reflecting 
with $\eta=.85$. Initial conditions are: $a=2.5 r_{0}(2.5$ times the largest asteroid semi-major axis), $e=.1$, $i=\pi / 2, \omega=0, \lambda=\pi / 2$, and time $t=1 e 7$ seconds. The model for Ida assumes $\mu=0.03 \mathrm{~km}^{3} / \mathrm{s}^{2}$ and $J_{2}=283.5 \mathrm{~km}^{2}$, computed by

$J_{2}=\left(a^{2}+b^{2}-2 c^{2}\right) / 10$

where $a=58 \mathrm{~km}, b=23 \mathrm{~km}$, and $c=23 \mathrm{~km}$ are the semi-major axes of Ida's shape. Thus we explicitly see that stable orbits about oblate spheroids are possible with imperfectly reflecting sails.

General and secular perturbation potentials

If we express the gravitational and SRP forces as potential forces acting on the spacecraft, we can compute the averaged effects on the orbital elements.

These potential forces are given by ${ }^{3,6}$

$\mathcal{R}_{g}=\frac{\mu J_{2}}{2 r^{3}}\left[3\left(\hat{\mathbf{p}}_{z} \cdot \mathbf{r}_{s}\right)^{2}-1\right]$

where $\mathcal{R}_{g}$ is the gravitational potential, and

$\mathcal{R}_{s r p}=a_{n p} r[\cos u \cos \lambda-\sin u \sin \lambda \cos i]$

where $\mathcal{R}_{\text {srp }}$ is the SRP force potential. $\lambda=\Omega-\nu_{\text {ast }}$ is the longitude of ascending node of the sail orbit relative to the sun-asteroid line and $\nu_{\text {ast }}$ is the true anomaly of the asteroid orbit. Together, the entire force potential for a solar sail at an oblate asteroid is $\mathcal{R}=\mathcal{R}_{g}+\mathcal{R}_{s r p}$. This formulation assumes that the sail is face-on to the sun.

The combined force potential can be averaged over the sail mean anomaly to find the secular potential

$R_{s}=\frac{1}{2 \pi} \int_{0}^{2 \pi} \mathcal{R} d M$

For the combined problem of SRP and oblate spheroid $^{3,6}$ the secular potential is

$$
\begin{aligned}
R_{s}= & \frac{\mu J_{2}}{4 a^{3}\left(1-e^{2}\right)^{3 / 2}}\left(1-3 W_{1}\right) \\
& -\frac{3}{2} a_{n p} a e W_{2} \\
W_{1}= & \cos ^{2} i \sin ^{2} \delta+\frac{1}{2} \sin 2 i \sin 2 \delta \sin \kappa \\
& +\sin ^{2} i \cos ^{2} \delta \sin ^{2} \kappa \\
W_{2}= & \cos \omega \cos \lambda-\sin \omega \sin \lambda \cos i
\end{aligned}
$$

where $a$ is the orbit semi-major axis, $e$ is the orbit eccentricity, and $\kappa=\Omega-\tilde{\alpha}=\lambda-\tilde{\alpha}+\nu_{a s t}$.
Given a perturbing potential of the form $U$, the Lagrange planetary equations with respect to time $\operatorname{are}^{4}$

$$
\begin{aligned}
\frac{d a}{d t} & =\frac{2}{n a} \frac{\partial U}{\partial M_{0}} \\
\frac{d e}{d t} & =\frac{1-e^{2}}{n a^{2} e} \frac{\partial U}{\partial M_{0}}-\frac{\sqrt{1-e^{2}}}{n a^{2} e} \frac{\partial U}{\partial \omega} \\
\frac{d i}{d t} & =\frac{\cot i}{n a^{2} \sqrt{1-e^{2}}} \frac{\partial U}{\partial \omega}-\frac{\csc i}{n a^{2} \sqrt{1-e^{2}}} \frac{\partial U}{\partial \Omega} \\
\frac{d M_{0}}{d t} & =-\frac{2}{n a} \frac{\partial U}{\partial a}-\frac{1-e^{2}}{n a^{2} e} \frac{\partial U}{\partial e} \\
\frac{d \omega}{d t} & =\frac{\sqrt{1-e^{2}}}{n a^{2} e} \frac{\partial U}{\partial e}-\frac{\cot i}{n a^{2} \sqrt{1-e^{2}}} \frac{\partial U}{\partial i} \\
\frac{d \Omega}{d t} & =\frac{\csc i}{n a^{2} \sqrt{1-e^{2}}} \frac{\partial U}{\partial i}
\end{aligned}
$$

For the secular potential $R_{s}$ we find the partials to be:

$$
\begin{aligned}
& \frac{\partial R_{s}}{\partial M_{0}}=0 \\
& \frac{\partial R_{s}}{\partial a}=-\frac{3 \mu J_{2}}{4 a^{4}\left(1-e^{2}\right)^{3 / 2}}\left(1-3 W_{3}\right)-\frac{3}{2} \times \\
& a_{n p} e[\cos \omega \cos \lambda-\sin \omega \sin \lambda \cos i] \\
& W_{3}=\cos ^{2} i \sin ^{2} \delta+\frac{1}{2} \sin 2 i \sin 2 \delta \sin \kappa \\
& +\sin ^{2} i \cos ^{2} \delta \sin ^{2} \kappa \\
& \frac{\partial R_{s}}{\partial e}=\frac{3 \mu J_{2} e}{4 a^{3}\left(1-e^{2}\right)^{5 / 2}}\left(1-3 W_{4}\right)-\frac{3}{2} \times \\
& a_{n p} a[\cos \omega \cos \lambda-\sin \omega \sin \lambda \cos i] \\
& W_{4}=\cos ^{2} i \sin ^{2} \delta+\frac{1}{2} \sin 2 i \sin 2 \delta \sin \kappa \\
& +\sin ^{2} i \cos ^{2} \delta \sin ^{2} \kappa \\
& \frac{\partial R_{s}}{\partial i}=\frac{3 \mu J_{2}}{4 a^{3}\left(1-e^{2}\right)^{3 / 2}} W_{5} \\
& -\frac{3}{2} a_{n p} \text { ae } \sin \omega \sin \lambda \sin i \\
& W_{5}=\sin 2 i \sin ^{2} \delta-\cos 2 i \sin 2 \delta \sin \kappa \\
& -\sin 2 i \cos ^{2} \delta \sin ^{2} \kappa \\
& \frac{\partial R_{s}}{\partial \omega}=\frac{3}{2} a_{n p} a e \times \\
& (\sin \omega \cos \lambda+\cos \omega \sin \lambda \cos i) \\
& \frac{\partial R_{s}}{\partial \Omega}=\frac{-3 \mu J_{2}}{4 a^{3}\left(1-e^{2}\right)^{3 / 2}} \sin i \cos \kappa W_{6}+\frac{3}{2} \times \\
& a_{n p} a e[\cos \omega \sin \lambda+\sin \omega \cos \lambda \cos i] \\
& W_{6}=\cos i \sin 2 \delta+2 \sin i \cos ^{2} \delta \sin \kappa
\end{aligned}
$$

Substituting these partials into the Lagrange equa- 
tions, we find the derivatives with respect to time as

$$
\begin{aligned}
& \frac{d a}{d t}=0 \\
& \frac{d e}{d t}=-\frac{3 a_{n p} \sqrt{1-e^{2}}}{2 n a} W_{7} \\
& W_{7}=\sin \omega \cos \lambda+\cos \omega \sin \lambda \cos i \\
& \frac{d i}{d t}=\frac{3 \mu J_{2}}{4 n a^{5}\left(1-e^{2}\right)^{2}} W_{8} \\
& -\frac{3 a_{n p} e}{2 n a \sqrt{1-e^{2}}} \cos \omega \sin \lambda \sin i \\
& W_{8}=\cos i \sin 2 \delta \cos \kappa+\sin i \cos ^{2} \delta \sin 2 \kappa \\
& \frac{d \omega}{d t}=\frac{3 \mu J_{2}}{4 n a^{5}\left(1-e^{2}\right)^{2}}\left(1-3 W_{9}\right) \\
& -\frac{3 \mu J_{2} \cot i}{4 n a^{5}\left(1-e^{2}\right)^{2}} W_{10}-\frac{3 a_{n p}}{2 n a} \times \\
& \frac{\sqrt{1-e^{2}}}{e}(\cos \omega \cos \lambda-\sin \omega \sin \lambda \cos i) \\
& +\frac{3 a_{n p}}{2 n a} \frac{e}{\sqrt{1-e^{2}}}(\sin \omega \sin \lambda \cos i) \\
& W_{9}=\cos ^{2} i \sin ^{2} \delta+\frac{1}{2} \sin 2 i \sin 2 \delta \sin \kappa \\
& +\sin ^{2} i \cos ^{2} \delta \sin ^{2} \kappa \\
& W_{10}=\sin 2 i \sin ^{2} \delta-\cos 2 i \sin 2 \delta \sin \kappa \\
& -\sin 2 i \cos ^{2} \delta \sin ^{2} \kappa \\
& \frac{d \Omega}{d t}=\frac{3 \mu J_{2} \csc i}{4 n a^{5}\left(1-e^{2}\right)^{2}} W_{11} \\
& -\frac{3 a_{n p} a e}{2 n a^{2} \sqrt{1-e^{2}}} \sin \omega \sin \lambda \\
& W_{11}=\sin 2 i \sin ^{2} \delta-\cos 2 i \sin 2 \delta \sin \kappa \\
& -\sin 2 i \cos ^{2} \delta \sin ^{2} \kappa
\end{aligned}
$$

The equations have their simplest form if we replace the independent variable of time with the asteroid orbit's true anomaly. Making this transformation yields:

$$
\begin{aligned}
\frac{d a}{d \nu_{a s t}}= & 0 \\
\frac{d e}{d \nu_{a s t}}= & -\Lambda \sqrt{1-e^{2}} \times \\
& (\sin \omega \cos \lambda+\cos \omega \sin \lambda \cos i) \\
\frac{d i}{d \nu_{a s t}}= & \frac{\Lambda \mu J_{2}}{2 P^{2} a^{2} a_{n p}} \times \\
& \left(\cos i \sin 2 \delta \cos \kappa+\sin i \cos ^{2} \delta \sin 2 \kappa\right) \\
& -\frac{\Lambda e}{\sqrt{1-e^{2}}} \cos \omega \sin \lambda \sin i \\
\frac{d \omega}{d \nu_{a s t}}= & \frac{\Lambda \mu J_{2}}{2 P^{2} a^{2} a_{n p}}\left(1-3 W_{12}\right) \\
& -\frac{\Lambda \mu J_{2} \cot i}{2 P^{2} a^{2} a_{n p}} W_{13}-\frac{\Lambda \sqrt{1-e^{2}}}{e} \times
\end{aligned}
$$

$$
\begin{aligned}
& (\cos \omega \cos \lambda-\sin \omega \sin \lambda \cos i) \\
& +\frac{e \Lambda}{\sqrt{1-e^{2}}} \sin \omega \sin \lambda \cos i \\
\frac{d \Omega}{d \nu_{a s t}}= & \frac{\Lambda \mu J_{2} \csc i}{2 P^{2} a^{2} a_{n p}} W_{13} \\
& -\frac{\Lambda e}{\sqrt{1-e^{2}}} \sin \omega \sin \lambda \\
\frac{d \lambda}{d \nu_{\text {ast }}}= & \frac{d \Omega}{d \nu_{\text {ast }}}-1
\end{aligned}
$$

where

$$
\begin{aligned}
W_{12}= & \cos ^{2} i \sin ^{2} \delta+\frac{1}{2} \sin 2 i \sin 2 \delta \sin \kappa \\
& +\sin ^{2} i \cos ^{2} \delta \sin ^{2} \kappa \\
W_{13}= & \sin 2 i \sin ^{2} \delta-\cos 2 i \sin 2 \delta \sin \kappa \\
& -\sin 2 i \cos ^{2} \delta \sin ^{2} \kappa \\
\Lambda= & \frac{3 a_{n p}}{2 \nu_{\text {ast }}^{\prime}} \sqrt{\frac{a}{\mu}} \\
= & \frac{3}{2} a_{\text {po }} R_{0}^{2} \sqrt{\frac{a}{P \mu \mu_{\text {sun }}}}
\end{aligned}
$$

and $P$ is the asteroid orbit parameter. The parameter $\Lambda$ is a measure of the solar radiation pressure perturbation. It is important to note that this parameter is a constant even if the asteroid is on an elliptic orbit. For this final set of equations the time varying term, $\kappa$, increases uniformly with true anomaly. These equations have been studied previously for the case $J_{2}=0^{1,6,7}$.

\section{Frozen Orbits with and without $J_{2}$}

An equilibrium point of the averaged Lagrange equations exists when all of the differential equations equal 0 . At this point there is no change, on average, to the orbital elements. These equilibrium solutions are also called "frozen orbits."

If $J_{2}$ is set to 0 , as in the case of a point-mass asteroid, then an equilibrium point for the averaged system has been found ${ }^{1}$ with $e_{0}=\sqrt{1 /\left(1+\Lambda^{2}\right)}$, $\lambda_{0}= \pm \pi / 2, i_{0}=\pi / 2$ and $\omega_{0}=\mp \pi / 2$.

These orbits are sun-synchronous and have a constant semi-major axis on average. They lie in the plane perpendicular to the asteroid-sun line and nominally have their periapsis aligned 90 degrees above or below the orbit plane. They depend on limiting the maximum acceleration of the sail according to (21) and limiting distance from the surface according to (22).

If we assume the asteroid's $J_{2}$ parameter to be small, or if the sail is sufficiently far from the asteroid, the $J_{2}$ terms will act as a perturbation on the 
sail orbit and will provide the time-varying forcing:

$$
\begin{aligned}
\frac{d a_{0}}{d \nu_{a s t}} & =0 \\
\frac{d e_{0}}{d \nu_{a s t}} & =0 \\
\frac{d \lambda_{0}}{d \nu_{a s t}} & =\frac{\Lambda \mu J_{2}}{2 P^{2} a^{2} a_{n p}} \sin 2 \delta \sin \kappa \\
\frac{d i_{0}}{d \nu_{a s t}} & =\frac{\Lambda \mu J_{2}}{2 P^{2} a^{2} a_{n p}} \cos ^{2} \delta \sin 2 \kappa \\
\frac{d \omega_{0}}{d \nu_{a s t}} & =\frac{\Lambda \mu J_{2}}{2 P^{2} a^{2} a_{n p}}\left(1-3 \cos ^{2} \delta \sin ^{2} \kappa\right)
\end{aligned}
$$

Thus we are left with a series of perturbations acting on $i, \omega$ and $\lambda$ which depend on $\kappa$ and $\delta$. If $\delta=\pi / 2$ then the rotation pole of the asteroid is aligned with the asteroid orbit angular momentum. In this case, $i$ and $\lambda$ are constant and $\omega$ increases at a constant rate. This is what we normally find for a polar orbit about an oblate planet. In general, however, $\delta$ will not be $\pi / 2$.

\section{The linear solution and its stability}

We can assess the dynamics and stability of the system if we linearize the equations for the orbital elements about the equilibrium point $\left(e_{0}=\right.$ $1 / \sqrt{1+\Lambda^{2}}, i_{0}=\pi / 2, \lambda_{0}= \pm \pi / 2$ and $\omega_{0}=\mp \pi / 2$ ).

After finding each of the partial derivatives and evaluating them, we assume that $J_{2}$ is small and of the same order as $\delta e, \delta \lambda$, etc. Neglecting products of these terms, we find the linearized system equations to be:

$$
\begin{aligned}
\frac{d \delta e}{d \nu_{a s t}}= & -\frac{\Lambda^{2}}{\sqrt{1+\Lambda^{2}}} \delta \lambda \\
\frac{d \delta \lambda}{d \nu_{a s t}}= & \frac{\Lambda \mu J_{2}}{2 P^{2} a^{2} a_{n p}} \sin 2 \delta \sin \kappa \\
& +\frac{\left(1+\Lambda^{2}\right)^{3 / 2}}{\Lambda^{2}} \delta e \\
\frac{d \delta i}{d \nu_{a s t}=} & \frac{\Lambda \mu J_{2}}{2 P^{2} a^{2} a_{n p}} \cos ^{2} \delta \sin 2 \kappa-\delta \omega \\
\frac{d \delta \omega}{d \nu_{a s t}}= & \frac{\Lambda \mu J_{2}}{2 P^{2} a^{2} a_{n p}}\left(1-3 \cos ^{2} \delta \sin ^{2} \kappa\right) \\
& +\left(1+\Lambda^{2}\right) \delta i
\end{aligned}
$$

We can rewrite these equations in matrix form as $d \delta \mathbf{x} / d \nu_{\text {ast }}=A \delta \mathbf{x}\left(\nu_{\text {ast }}\right)+B\left(\nu_{\text {ast }}\right)$ where

$$
A=\left[\begin{array}{cccc}
0 & -\frac{\Lambda^{2}}{\sqrt{1+\Lambda^{2}}} & 0 & 0 \\
\frac{\left(1+\Lambda^{2}\right)^{3 / 2}}{\Lambda^{2}} & 0 & 0 & 0 \\
0 & 0 & 0 & -1 \\
0 & 0 & 1+\Lambda^{2} & 0
\end{array}\right]
$$

and

$$
B=\frac{\Lambda \mu J_{2}}{2 P^{2} a^{2} a_{n p}}\left[\begin{array}{c}
0 \\
\sin 2 \delta \sin \kappa \\
\cos ^{2} \delta \sin ^{2} \kappa \\
1-3 \cos ^{2} \delta \sin ^{2} \kappa
\end{array}\right]
$$

Since $A$ is constant in $\nu_{a s t}$, we can use the state transition matrix, $\boldsymbol{\Phi}\left(\nu_{\text {ast }}, v\right)=\mathcal{L}^{-1}\left\{(s I-A)^{-1}\right\}$ to help solve for $\mathbf{x}\left(\nu_{\text {ast }}\right)$. We find,

$$
\begin{aligned}
& \Phi(\tilde{\nu})=(76) \\
& {\left[\begin{array}{ccc}
\cos w_{2} \tilde{\nu} & -\left(\frac{\Lambda}{w_{2}}\right)^{2} \sin w_{2} \tilde{\nu} & \\
\left(\frac{w_{2}}{\Lambda}\right)^{2} \sin w_{2} \tilde{\nu} & \cos w_{2} \tilde{\nu} & \ldots \\
0 & 0 & \\
0 & 0 &
\end{array}\right]} \\
& {\left[\begin{array}{ccc} 
& 0 & 0 \\
\cdots & 0 & 0 \\
& \cos w_{2} \tilde{\nu} & -\frac{\sin w_{2} \tilde{\nu}}{w_{2}} \\
& w_{2} \sin w_{2} \tilde{\nu} & \cos w_{2} \tilde{\nu}
\end{array}\right]}
\end{aligned}
$$

where $\tilde{\nu}=\nu_{a s t}-v$ and $w_{2}=\sqrt{1+\Lambda^{2}}$. For a timeforced system,

$$
\begin{aligned}
\delta \mathbf{x}\left(\nu_{a s t}\right)= & \boldsymbol{\Phi}\left(\nu_{a s t}, 0\right) \delta \mathbf{x}_{i}+ \\
& \int_{0}^{\nu_{a s t}} \boldsymbol{\Phi}\left(\nu_{a s t}, v\right) B(v) u(v) d v
\end{aligned}
$$

where $\delta \mathbf{x}_{i}=\left[\begin{array}{llll}\delta e_{i} & \delta \lambda_{i} & \delta i_{i} & \delta \omega_{i}\end{array}\right]^{T}$ are the initial conditions.

In our case the entire solution $\delta \mathbf{x}\left(\nu_{\text {ast }}\right)$ is:

$$
\begin{aligned}
& \delta \mathbf{x}\left(\nu_{\text {ast }}\right)= \\
& {\left[\begin{array}{c}
\delta e_{i} \cos w_{2} \nu_{a s t}-\delta \lambda_{i}\left(\frac{\Lambda}{w_{2}}\right)^{2} \sin w_{2} \nu_{a s t} \\
\delta e_{i}\left(\frac{\Lambda}{w_{2}}\right)^{2} \sin w_{2} \nu_{a s t}+\delta \lambda_{i} \cos w_{2} \nu_{a s t} \\
\delta i_{i} \cos w_{2} \nu_{a s t}-\frac{\delta \omega_{i}}{w_{2}} \sin w_{2} \nu_{a s t} \\
w_{2} \delta i_{i} \sin w_{2} \nu_{a s t}+\delta \omega_{i} \cos w_{2} \nu_{a s t}
\end{array}\right]} \\
& +\frac{\Lambda \mu J_{2}}{2 P^{2} a^{2} a_{n p}}\left[\begin{array}{c}
-\left(\frac{\Lambda}{w_{2}}\right)^{2} \mathcal{C} \sin 2 \delta \\
\mathcal{F} \sin 2 \delta \\
\mathcal{M} \cos ^{2} \delta+\frac{3}{w_{2}} \mathcal{N} \cos ^{2} \delta- \\
\frac{1}{w_{2}^{2}}\left(1-\cos w_{2} \nu_{\text {ast }}\right) \\
w_{2} \mathcal{N} \cos ^{2} \delta-3 \mathcal{M} \cos ^{2} \delta+ \\
\frac{\sin w_{2} \nu_{a s t}}{w_{2}} \times \\
\left(2 \cos w_{2} \nu_{\text {ast }}-1\right)
\end{array}\right]
\end{aligned}
$$

where $\mathcal{C}, \mathcal{F}, \mathcal{M}$ and $\mathcal{N}$ are given in Appendix A. 
There is a resonance singularity within these equations if $w_{2}=2($ or $\Lambda=\sqrt{3})$, denoting a semi-major axis of

$a_{\text {res }}=\frac{4}{3} \frac{a_{\text {ast }} \mu \mu_{\text {sun }}}{a_{\text {po }}^{2} R_{0}^{4}}$

This singularity is usually not a problem, however, since for this value to occur the sail orbit semi-major axis would have to be less than the asteroid radius in general $\left(a_{\text {res }}=1.21 \mathrm{~km}\right.$ for the orbits about Ida that we are using for examples in this paper.)

Linear continuous-time systems of the form:

$\dot{\mathbf{x}}=A(\nu) \mathbf{x}(\nu)+B(\nu) \mathbf{u}(\nu)$

are stable in the sense of Lyapunov if the norm of $\mathbf{x}$, $\|\mathbf{x}(\nu)\|$, is bounded for all $\nu \geq 0^{8}$.

Looking at the unforced case, $\mathbf{x}\left(\nu_{\text {ast }}\right)=$ $\Phi\left(\nu_{a s t}, 0\right) \mathbf{x}(0)$, we must show that

$$
\begin{aligned}
\left\|\mathbf{x}\left(\nu_{a s t}\right)\right\| & =\left\|\Phi\left(\nu_{a s t}, 0\right) \mathbf{x}(0)\right\| \\
& \leq\left\|\Phi\left(\nu_{a s t}, 0\right)\right\|\|\mathbf{x}(0)\|
\end{aligned}
$$

is bounded. Since the initial conditions are bounded, we only need to show that

$\left\|\Phi\left(\nu_{\text {ast }}, 0\right)\right\| \leq N$ for all $\nu_{\text {ast }} \geq 0$

The eigenvalues of $A$ are $\lambda_{\epsilon}= \pm \sqrt{1+\Lambda^{2}} i$ and $\pm \sqrt{1+\Lambda^{2}} i$. The eigenvalues are repeated, however, the two sets of conjugate pairs are not coupled so the unforced systems are Lyapunov stable. There is, therefore, a number $N$ such that (84) is satisfied.

For the forced case, we need to show that $\left\|\mathbf{x}\left(\nu_{\text {ast }}\right)\right\|$ is bounded by some other number $M$. Since we know that the homogeneous solution is bounded, we only need to show that,

$\left\|\int_{0}^{\nu_{a s t}} \Phi\left(\nu_{a s t}, v\right) B(v) u(v) d v\right\| \leq M$

Since $0 \leq \nu_{\text {ast }}<2 \pi$, we know that,

$$
\begin{gathered}
\left\|\int_{0}^{\nu_{a s t}} \Phi\left(\nu_{a s t}, v\right) B(v) u(v) d v\right\| \leq \\
\left\|\int_{0}^{2 \pi} \Phi\left(\nu_{a s t}, v\right) B(v) u(v) d v\right\|
\end{gathered}
$$

Therefore, the forced case is stable as well.

$\underline{\text { Non-linear Stability }}$

Although we find the orbits to be stable in theory, there are some parameter combinations which lead to non-linear instabilities. For example, if the semimajor axis of the sail orbit is small (within two asteroid radii), the eccentricity can grow large (more than $.5)$. When this situation occurs, the orbit periapsis can become less than one asteroid radius (according to the equation $r_{p}=a(1-e)$ ) and the spacecraft may impact on the surface of the asteroid.

Due to the $J_{2}$ perturbations, the SRP solution oscillates about the ideal fixed point, meaning that all the orbit elements take on a range of values. When the amplitude of these oscillations is large enough, the linearized equations of motion do not apply anymore and the dynamics become dominated by the closed form solution for the averaged SRP force ${ }^{1}$. This situation is exemplified in the series of computations presented in Figures 6-15, showing the orbit dynamics of a sail about an asteroid with and without a $J_{2}$ gravity term. In the absence of the $J_{2}$ term the orbit is stable, however the addition of $J_{2}$ causes the eccentricity to rise to extremely large values over short time spans.

This instability is non-linear in general and is closely related to the original solution of the Lagrange equations for the SRP-only case. As reported in Morrow et al. ${ }^{1}$ the solution for eccentricity in the SRP-only case, with an initial value for eccentricity of zero, is:

$$
\begin{aligned}
e_{S R P}= & \sqrt{1-\sin ^{2} i_{0} \sin ^{2} \lambda_{0}} \times \\
& \sin \left(\nu_{\text {ast }} \sqrt{1+\Lambda^{2}}\right)
\end{aligned}
$$

where $i_{0}$ and $\lambda_{0}$ are the initial values of these angles. From this condition we see that deviations in the initial values of these angles can excite the eccentricity to reach maximum values of $\sqrt{1-\sin ^{2} i_{0} \sin ^{2} \lambda_{0}}$ at a later time. For our frozen orbits we nominally set these angles equal to $90^{\circ}$, but perturbations from $J_{2}$ give them, essentially, initial conditions that deviate from these angles. Adding this non-linear effect to the natural oscillation of $e$ due to $J_{2}$ and the forced oscillations in the other orbit elements can lead to large deviations in eccentricity over relatively short time spans, as is apparent in the figures.

Thus, while previous results indicated that frozen sail orbits close to the asteroid tended to become more robust, here we see that asteroid shape effects can destabilize motion when close to the asteroid. Thus, a compromise must be struck between for feasible sail operations about an asteroid.

Figures 6 and 7 are trajectories shown in the frame aligned with the asteroid rotation pole. We use this coordinate system because it is easier to see the degradation to the orbit under the $J_{2}$ perturbation. In Figure 6, with $J_{2}=0$, the orbit stays fixed as expected. In Figure 7 , with $J_{2} \neq 0$, the orbit becomes elongated and skewed, the periapsis radius becomes smaller, and the spacecraft eventually crashes into the asteroid surface. 


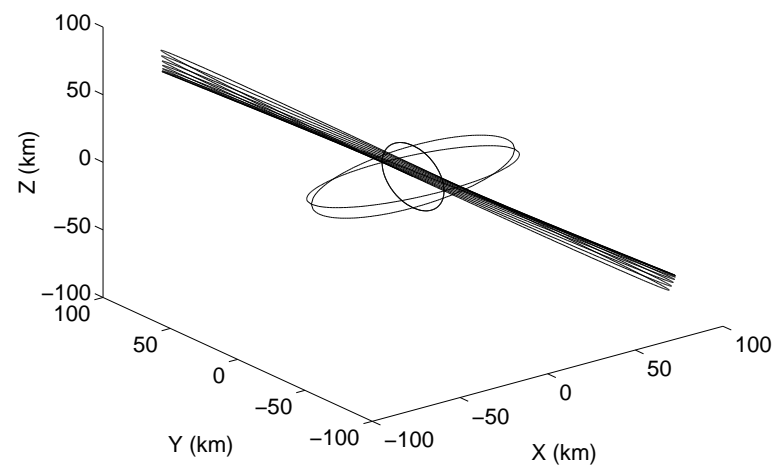

Figure 6: Orbit about Ida with tilted asteroid rotation axis, with $J_{2}=0$. Initial conditions are: $a=$ $116 \mathrm{~km}$ (two times the largest asteroid semi-major axis), $e=.1, i=\pi / 2, \omega=0, \Omega=\pi / 2$, and $\nu=0$; and time $t=3.2 \mathrm{e} 5 \mathrm{~s}$.

Figures 8 - 15 show plots of the orbital elements with and without $J_{2}$. We used an altitude of $2.5 r_{0}$ for these plots which does not crash within the time interval plotted, but clearly shows the perturbing effect of $J_{2}$.

\section{Conclusion}

In the current study, we have shown that hovering solutions and orbital options still exist under the relaxed assumptions made to our model.

The volume of space surrounding a point-mass asteroid where hovering points exist has been reduced by allowing for an imperfectly reflecting sail, however, there are still a continuum of hovering positions available.

When perturbations due to asteroid oblateness are included, we find new constraints on feasible orbits. However, a large family of stable and sun synchronous orbits still exist. We have shown that distance from the asteroid is a major factor in finding feasible orbits about oblate asteroids. We must allow for the spacecraft to be neither too far away nor too near. Within this "altitude envelope," however, good coverage of the asteroid is available for extended periods of time.

For long-duration missions to asteroids, solar sails have the advantage over conventional spacecraft of having an unlimited fuel supply from sunlight. They are capable of rendezvous with an asteroid then escaping from orbit to meet another planetary body or returning to Earth.

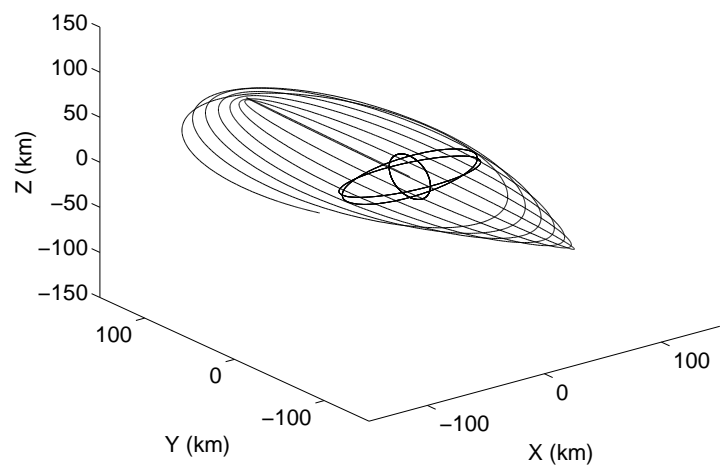

Figure 7: Orbit about Ida in the rotating frame with $J_{2} \neq 0$. All other conditions the same as in figure 6 . In this figure we can see that $a$ stays constant while $e$ changes so that the periapsis radius grows smaller. The spacecraft will eventually intersect the asteroid.

\section{Acknowledgements}

Research at the University of California, San Diego, was supported by the National Science Foundation NSF-OPP 98 06941. Research at The University of Michigan was supported by the IPN Technology Program by a grant from the Jet Propulsion Laboratory, California Institute of Technology which is under contract with the National Aeronautics and Space Administration.

\section{Bibliography}

[1] Morrow, E., D. J. Scheeres, D. Lubin. 2000. "Solar Sail Orbit Operations at Asteroids," Journal of Spacecraft and Rockets, Vol. 38, No. 2, 279-286.

[2] McInnes, C.R. 1998. "Artificial Lagrange Points for a Partially Reflecting Flat Solar Sail," Journal of Guidance, Vol. 22, No. 1, 185-187.

[3] Scheeres, D.J., S. J. Ostro, R. S. Hudson, E. M. DeJong, S. Suzuki. 1998. "Dynamics of Orbits Close to Asteroid 4179 Toutatis," Icarus, Vol. 132, Article No. IS975870, 53-79.

[4] Danby, J.M.A. 1988. Fundamentals of Celestial Mechanics. Willmann-Bell, Inc., Richmond, VA

[5] McInnes, C.R. 1999. Solar Sailing: Technology, Dynamics and Mission Applications. Praxis Publishing Ltd, Chichester, UK

[6] Scheeres, D.J. 1999. "Satellite Dynamics about Small Bodies: Averaged Solar Radiation Pressure Effects," Journal of the Astronautical Sciences, Vol. textit47, Nos. 1 and 2, 25-46. 


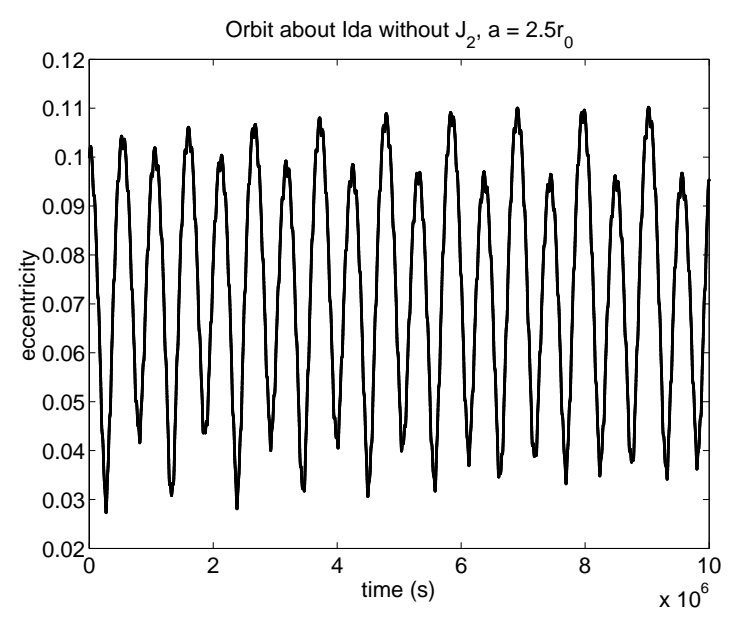

Figure 8: Eccentricity vs. time when $J_{2}=0$.

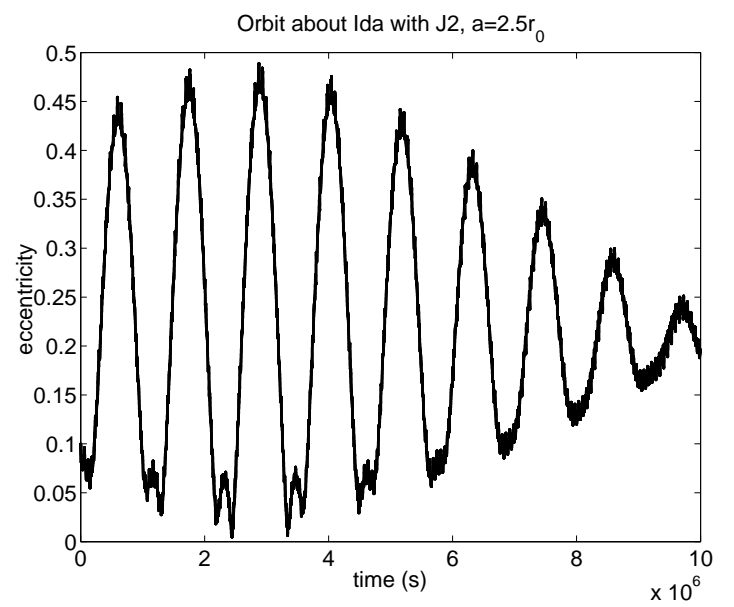

Figure 9: Eccentricity vs. time when $J_{2} \neq 0$.

[7] Mignard, F. and Hénon, M., "About an Unsuspected Integrable Problem," Celestial Mechanics, Vol. 33, 1984, pp. $239-250$.

[8] Brogan, W.L. 1991. Modern Control Theory. Prentice-Hall, Inc., Englewood Cliffs, New Jersey

\section{Appendix A}

Coefficients used in the linearized solution

Note that in this appendix, $\nu$ is the asteroid true anomaly.

$$
\begin{aligned}
\mathcal{C}= & \sin \left(w_{2} \nu-\lambda_{0}+\tilde{\alpha}\right)\left[\frac{1-\cos \left(w_{2}+1\right) \nu}{2\left(w_{2}+1\right)}\right] \\
& -\sin \left(w_{2} \nu+\lambda_{0}-\tilde{\alpha}\right)\left[\frac{1-\cos \left(w_{2}-1\right) \nu}{2\left(w_{2}-1\right)}\right] \\
& +\cos \left(w_{2} \nu-\lambda_{0}+\tilde{\alpha}\right)\left[\frac{\sin \left(w_{2}+1\right) \nu}{2\left(w_{2}+1\right)}\right]
\end{aligned}
$$

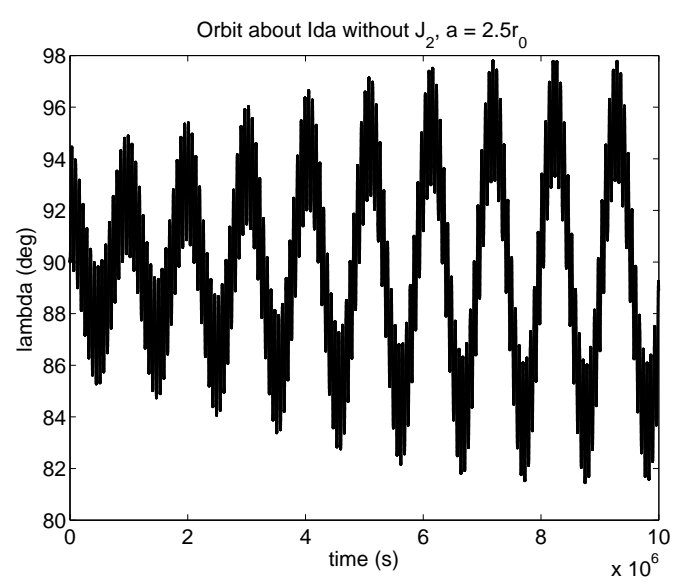

Figure 10: $\lambda$ vs. time when $J_{2}=0$.

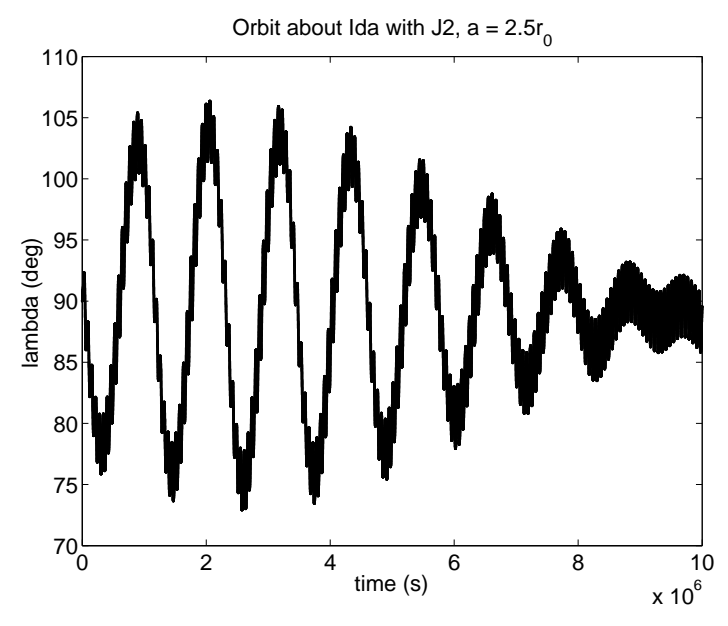

Figure 11: $\lambda$ vs. time when $J_{2} \neq 0$.

$$
-\cos \left(w_{2} \nu+\lambda_{0}-\tilde{\alpha}\right)\left[\frac{\sin \left(w_{2}-1\right) \nu}{2\left(w_{2}-1\right)}\right]
$$

$$
\begin{aligned}
\mathcal{F}= & \cos \left(w_{2} \nu-\lambda_{0}+\tilde{\alpha}\right)\left[\frac{1-\cos \left(w_{2}+1\right) \nu}{2\left(w_{2}+1\right)}\right] \\
& -\cos \left(w_{2} \nu+\lambda_{0}-\tilde{\alpha}\right)\left[\frac{1-\cos \left(w_{2}-1\right) \nu}{2\left(w_{2}-1\right)}\right] \\
& +\sin \left(w_{2} \nu+\lambda_{0}-\tilde{\alpha}\right)\left[\frac{\sin \left(w_{2}-1\right) \nu}{2\left(w_{2}-1\right)}\right] \\
& -\sin \left(w_{2} \nu-\lambda_{0}+\tilde{\alpha}\right)\left[\frac{\sin \left(w_{2}+1\right) \nu}{2\left(w_{2}+1\right)}\right] \\
\mathcal{M}= & \mathcal{G} \cos w_{2} \nu \sin ^{2}\left(\lambda_{0}-\tilde{\alpha}\right)+ \\
& \mathcal{H} \cos w_{2} \nu \sin ^{2}\left(\lambda_{0}-\tilde{\alpha}\right) \cos \left(\lambda_{0}-\tilde{\alpha}\right)+ \\
& \mathcal{I} \cos w_{2} \nu \cos ^{2}\left(\lambda_{0}-\tilde{\alpha}\right)+ \\
& \mathcal{J} \sin w_{2} \nu \sin ^{2}\left(\lambda_{0}-\tilde{\alpha}\right)+
\end{aligned}
$$




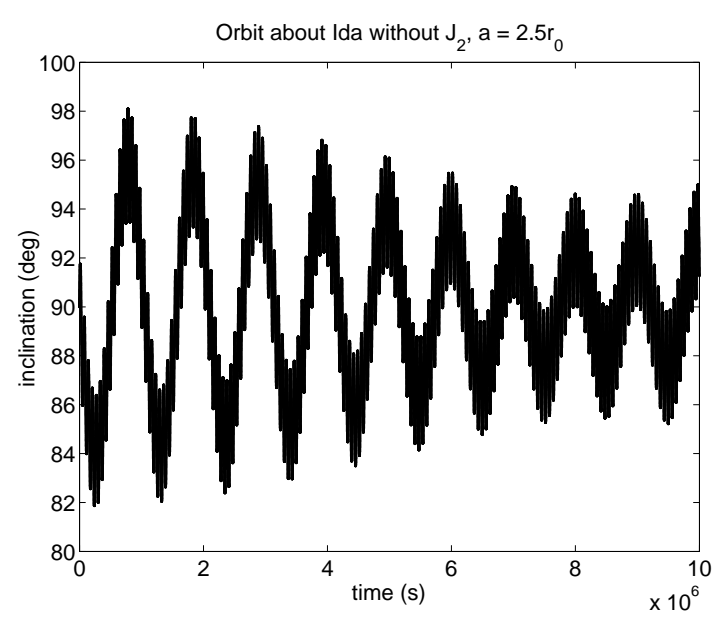

Figure 12: Inclination vs. time when $J_{2}=0$.

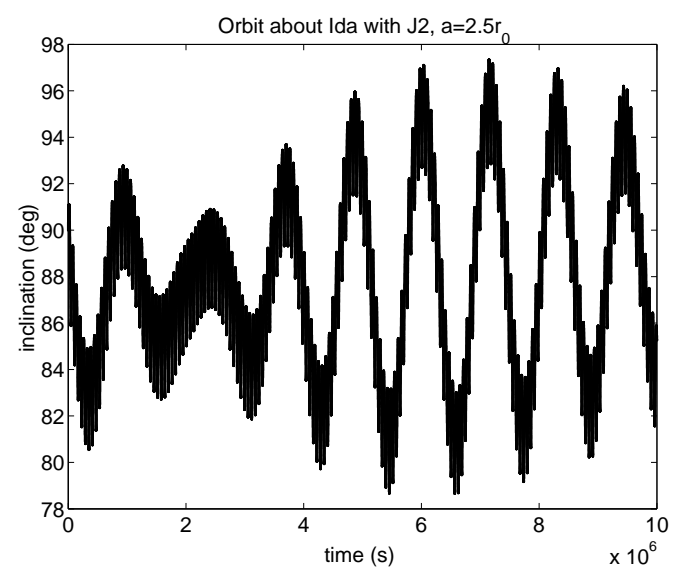

Figure 13: Inclination vs. time when $J_{2} \neq 0$.

$$
\begin{aligned}
& \mathcal{K} \sin w_{2} \nu \sin \left(\lambda_{0}-\tilde{\alpha}\right) \cos \left(\lambda_{0}-\tilde{\alpha}\right)+ \\
& \mathcal{L} \sin w_{2} \nu \cos ^{2}\left(\lambda_{0}-\tilde{\alpha}\right) \\
\mathcal{N}= & \mathcal{G} \sin w_{2} \nu \sin ^{2}\left(\lambda_{0}-\tilde{\alpha}\right)+ \\
& \mathcal{H} \sin w_{2} \nu \sin \left(\lambda_{0}-\tilde{\alpha}\right) \cos \left(\lambda_{0}-\tilde{\alpha}\right)+ \\
& \mathcal{I} \sin w_{2} \nu \cos ^{2}\left(\lambda_{0}-\tilde{\alpha}\right)- \\
& \mathcal{J} \cos w_{2} \nu \sin ^{2}\left(\lambda_{0}-\tilde{\alpha}\right)- \\
& \mathcal{K} \cos w_{2} \nu \sin \left(\lambda_{0}-\tilde{\alpha}\right) \cos \left(\lambda_{0}-\tilde{\alpha}\right)- \\
& \mathcal{L} \cos w_{2} \nu \cos ^{2}\left(\lambda_{0}-\tilde{\alpha}\right)
\end{aligned}
$$

where

$$
\begin{array}{r}
\mathcal{G}=\frac{1}{2 w_{2}} \sin w_{2} \nu+\frac{1}{4} \times \\
{\left[\frac{\sin \left(w_{2}-2\right) \nu}{w_{2}-2}+\frac{\sin \left(w_{2}+2\right) \nu}{w_{2}+2}\right]} \\
\mathcal{H}=\frac{1}{2} \times
\end{array}
$$

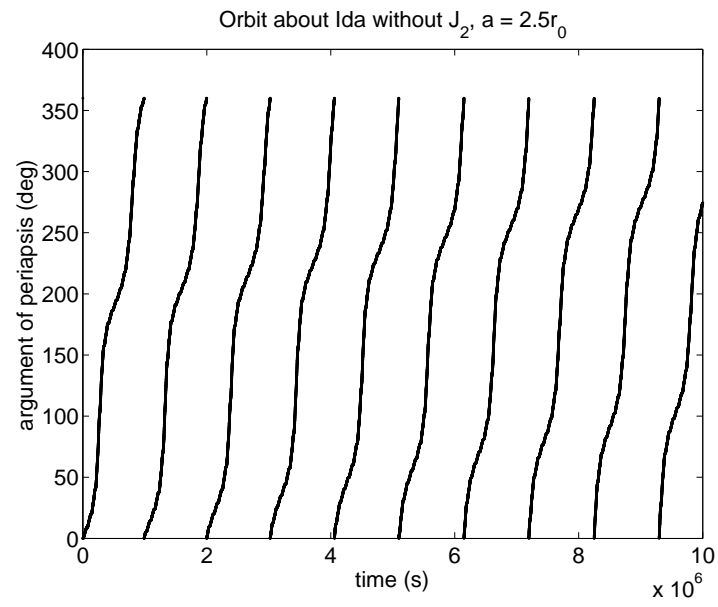

Figure 14: Argument of periapsis vs. time, $J_{2}=0$.

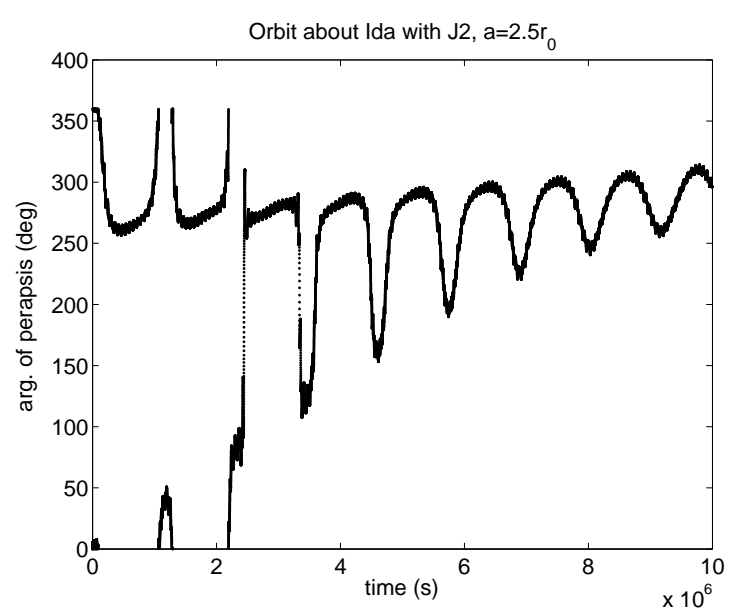

Figure 15: Argument of periapsis vs. time, $J_{2} \neq 0$.

$$
\begin{array}{r}
{\left[\frac{1-\cos \left(w_{2}+2\right) \nu}{w_{2}+2}-\frac{1-\cos \left(w_{2}-2\right) \nu}{w_{2}-2}\right]} \\
\mathcal{I}=\frac{1}{2 w_{2}} \sin w_{2} \nu-\frac{1}{4} \times \\
{\left[\frac{\sin \left(w_{2}-2\right) \nu}{w_{2}-2}+\frac{\sin \left(w_{2}+2\right) \nu}{w_{2}+2}\right]} \\
\mathcal{J}=\frac{1}{2 w_{2}}\left[1-\cos w_{2} \nu\right]+\frac{1}{4} \times \\
{\left[\frac{1-\cos \left(w_{2}+2\right) \nu}{w_{2}+2}+\frac{1-\cos \left(w_{2}-2\right) \nu}{w_{2}-2}\right]} \\
\mathcal{K}=\frac{1}{2}\left[\frac{\sin \left(w_{2}-2\right) \nu}{w_{2}-2}-\frac{\sin \left(w_{2}+2\right) \nu}{w_{2}+2}\right] \\
\mathcal{L}=\frac{1}{2 w_{2}}\left[1-\cos w_{2} \nu\right]-\frac{1}{4} \times \\
{\left[\frac{1-\cos \left(w_{2}+2\right) \nu}{w_{2}+2}+\frac{1-\cos \left(w_{2}-2\right) \nu}{w_{2}-2}\right]}
\end{array}
$$

